\title{
Observing Changes in Ocean Carbonate Chemistry: Our Autonomous Future
}

\author{
Seth M. Bushinsky ${ }^{1}\left[\right.$ ] $\cdot$ Yuichiro Takeshita ${ }^{2} \cdot$ Nancy L. Williams $^{3}$ \\ Published online: 7 May 2019 \\ (C) The Author(s) 2019
}

\begin{abstract}
Purpose of Review We summarize recent progress on autonomous observations of ocean carbonate chemistry and the development of a network of sensors capable of observing carbonate processes at multiple temporal and spatial scales.

Recent Findings The development of versatile $\mathrm{pH}$ sensors suitable for both deployment on autonomous vehicles and in compact, fixed ecosystem observatories has been a major development in the field. The initial large-scale deployment of profiling floats equipped with these new $\mathrm{pH}$ sensors in the Southern Ocean has demonstrated the feasibility of a global autonomous open-ocean carbonate observing system.

Summary Our developing network of autonomous carbonate observations is currently targeted at surface ocean $\mathrm{CO}_{2}$ fluxes and compact ecosystem observatories. New integration of developed sensors on gliders and surface vehicles will increase our coastal and regional observational capability. Most autonomous platforms observe a single carbonate parameter, which leaves us reliant on the use of empirical relationships to constrain the rest of the carbonate system. Sensors now in development promise the ability to observe multiple carbonate system parameters from a range of vehicles in the near future.
\end{abstract}

Keywords Autonomous platforms · Carbonate observations · Ocean acidification · Ocean biogeochemical sensors

\section{Introduction}

The oceanic carbonate system is going through unprecedented change. Each year, the ocean absorbs approximately $25 \%$ of anthropogenic emissions of carbon dioxide $\left(\mathrm{CO}_{2}\right)$ to the atmosphere [1] and has absorbed at least $25 \%$ of all anthropogenic $\mathrm{CO}_{2}$ since the industrial revolution $[2,3]$. While this reduces atmospheric $\mathrm{CO}_{2}$ concentrations, it comes at a cost.

This article is part of the Topical Collection on Carbon Cycle and Climate

Seth M. Bushinsky

sb17@princeton.edu

1 Program in Atmospheric and Oceanic Sciences, Princeton University, 300 Forrestal Road, Sayre Hall, Princeton, NJ 08544, USA

2 Monterey Bay Aquarium Research Institute, 7700 Sandholdt Road, Moss Landing, CA, USA

3 Pacific Marine Environmental Laboratory, National Oceanic and Atmospheric Administration, 7600 Sand Point Way, NE, Seattle, WA, USA
The dissolving of $\mathrm{CO}_{2}$ acidifies the seawater (lowers $\mathrm{pH}$ ) and shifts the equilibrium of carbonate species, decreasing carbonate ion and increasing bicarbonate concentration [4-6]. On average, open ocean $\mathrm{pH}$ has decreased by approximately 0.0018 year $^{-1}$ over the past $15-30$ years [7]. This process, known as ocean acidification, is happening more rapidly than at any other time in Earth's history [8].

Ocean acidification is thought to have widespread detrimental impacts on marine organisms and ecosystems including those that support valuable fisheries [9-11]. For example, pteropods, a pelagic sea snail that is an important prey species for fish such as salmon, cod, and mackerel, have been demonstrated to be especially vulnerable to elevated $\mathrm{CO}_{2}$ conditions [12]. Mass mortality events in shellfish hatcheries have also been linked to ocean acidification [13]. Coral reefs, which provide trillions of dollars in societal services worldwide, are projected to experience decreased net calcification, a key process in maintaining ecosystem function [14]. The impact of acidification is being felt globally, but with significant heterogeneity in the temporal and spatial patterns of response due to regional differences in chemistry, circulation, and biology. For example, modeling results predict that some ocean regions 
will acidify significantly faster than the open ocean, such as upwelling regions like the California Current System [15], the Arctic Ocean [16], and the Southern Ocean [17], making them potentially more vulnerable to ocean acidification. To observe predicted changes in the ocean carbonate system, we must have an instrumentation network that can capture acidification and its effects at multiple temporal and spatial scales.

There are four "master" variables for the marine carbonate system that we can measure: partial pressure (or fugacity) of $\mathrm{CO}_{2}\left(p \mathrm{CO}_{2}\right), \mathrm{pH}$, dissolved inorganic carbon (DIC), and total alkalinity (TA). The carbonate system can be described by a system of equations such that it can be fully constrained by measuring any two of the four parameters [4, 5]. Different combinations of parameters must be measured or calculated depending on the biogeochemical processes of interest. For example, $p \mathrm{CO}_{2}$ is required to study the air-sea $\mathrm{CO}_{2}$ flux, as the difference between air and seawater $p \mathrm{CO}_{2}$ describes the thermodynamic potential for $\mathrm{CO}_{2}$ to go in or out of the water [18]. DIC is particularly useful for studying production and respiration dynamics in the open ocean $[19,20]$. TA can be used to study calcification and dissolution processes. Anthropogenic carbon inventory calculations often use both DIC and TA [21].

Observing changes in ocean conditions on the spatiotemporal scales necessary to constrain carbon uptake, storage rates, and subsequent ecosystem impacts remains a significant challenge. Prior to the development of autonomous systems to measure ocean carbonate chemistry, our understanding of the ocean carbonate system has come largely from discrete measurements on repeat hydrography cruises that occupy transects across the ocean basins approximately every decade [21-23], monthly to seasonal time-series stations at single locations [7], and underway surface observations of $p \mathrm{CO}_{2}$ from research vessels and ships of opportunity [24]. These programs have provided invaluable knowledge such as the ability to quantify anthropogenic carbon inventories of the global ocean [2], mean annual oceanic air-sea $\mathrm{CO}_{2}$ flux [25], and open-ocean acidification rates [7]. However, there are limitations for shipbased observing strategies. For example, large areas of the ocean have never been sampled due to long transit times and the expensive operating costs of research vessels. Decadal observations provide no information on seasonal or interannual variability. Data can be skewed towards summer months, as research cruises are more frequently conducted during calmer months especially in regions like the Southern Ocean where harsh wintertime conditions make shipboard operations difficult. Capturing the dynamic spatiotemporal variability in coastal oceans can also be challenging from shipboard measurements. In order to meet future scientific and societal needs, development of new observational strategies is required.

The recent expansion of autonomous platforms such as moorings, profiling floats, underwater gliders, and mobile surface vehicles provides a scalable solution to this undersampling problem. Moorings are floating buoys anchored to the bottom of the ocean for typical deployment lengths of up to a year. Profiling floats are buoyancy-driven drifters that sample the water column on a regular cycle, staying in deep waters in between profiles to conserve battery power and limit bio-fouling [26]. Steerable profiling vehicles can be buoyancy-driven underwater gliders [27], or propellerdriven autonomous underwater vehicles [28], with tradeoffs between deployment length and speed. Mobile surface vehicles are relatively new tools for biogeochemical observations, powered by wind or wave energy to achieve comparatively fast speeds while carrying larger payloads than floats or gliders. While observations of $p \mathrm{CO}_{2}$ from moorings are robust and established, deployments of sensors that measure carbonate system parameters have only recently begun on other autonomous platforms. In particular, the recent addition of $\mathrm{pH}$ to profiling platforms has set the stage for revolutionary developments in this field. For example, as of January, 2019, over $10,000 \mathrm{pH}$ profiles (biogeochemical-argo.org) have been made from profiling floats since the first deployments in 2012. This is over twice the number of ship-based $\mathrm{pH}$ profiles spanning 1972-2013 [29]. Autonomous platforms and vehicles provide finer scale resolution and coverage than ships or moorings can provide and will form an important part of our future ocean carbonate observing system.

In order to distinguish long-term changes in processes from variations in the natural mean state, it is necessary to observe processes over the relevant temporal and spatial scales [30]. For instance, patterns of air-sea $\mathrm{CO}_{2}$ exchange are important to observe on short time and space scales in order to understand the gas exchange component of surface observations, but also must be measured globally over annual and decadal time scales to understand long-term changes in the oceanic uptake of anthropogenic $\mathrm{CO}_{2}$. Autonomous platforms inherently involve tradeoffs in lifetime, payload, power consumption, and measurement frequency, meaning that no single tool can adequately sample all of the various processes of interest (Fig. 1). For most processes, fully capturing the relevant scales of variability and change will require a mix of vehicles. For example, individual floats can be interpreted in a 1dimensional sense, providing detailed information on gas exchange and upper ocean processes, while an array of floats, moorings, and gliders can provide information on basin to global scales.

In this review, we summarize work from the past 5 years highlighting the expansion of our autonomous carbonate observing capabilities, and some of the key recent scientific discoveries. Recent reviews have highlighted advances in carbonate sensor technology [31, 32] so we instead focus on the autonomous instrumentation that currently comprises our carbonate observation network and promising emerging technologies. In this paper, we review (1) current autonomous 


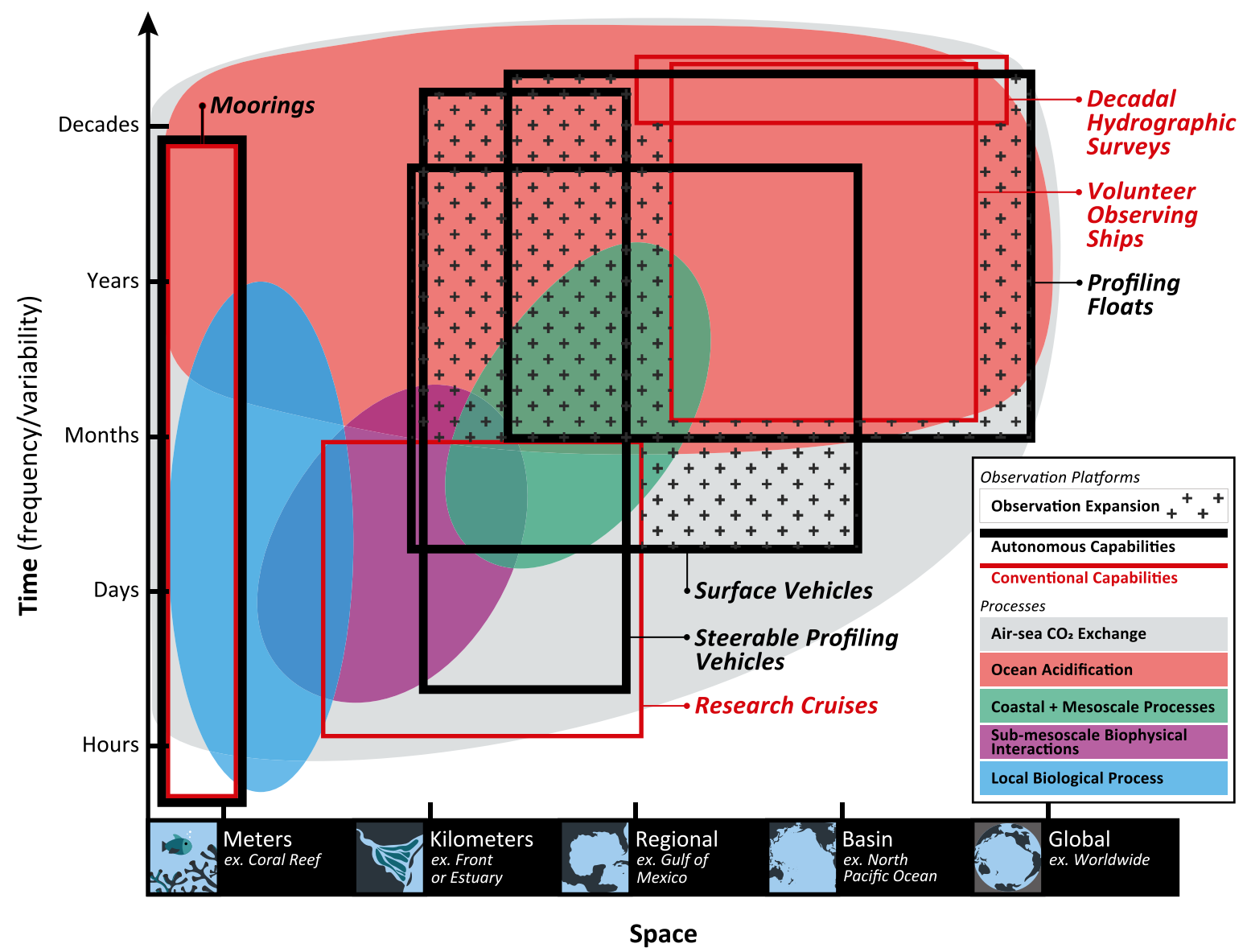

Fig. 1 Observational capabilities and carbonate system processes as a function of time and space. Ocean processes that affect the carbonate system (solid colored shapes with labels in the caption) are depicted as a function of the temporal and spatial scales over which they must be observed to capture important variability and/or long-term change. The ability of different platforms to capture carbonate system processes is overlaid for conventional approaches (red boxes, thinner lines) and autonomous arrays (black, thick lines). Not all observational platforms currently provide equivalent measurements capabilities, in terms of either parameters measured or spatial/temporal resolution. For example, profiling floats are only equipped with $\mathrm{pH}$ sensors at present, while the Volunteer Observing Ships provide only underway surface measurements. Furthermore, the capability of a given platform to provide long-

observational capabilities, (2) emerging sensors and autonomous platforms for carbonate observations, and (3) challenges for the measurement and interpretation of these novel observations.

\section{Current Autonomous Observational Capabilities}

\section{Surface $\mathrm{CO}_{2}$ Measurements}

Surface moorings are the oldest platform for autonomous observation of the carbonate system. Moorings provide highfrequency observations that can be extended for years to duration measurements is not entirely captured in this figure; a research cruise may provide a snapshot of a mesoscale process over several weeks, but does not typically capture that process repeatedly over time. The exact spatial and temporal sampling area covered by each platform will change as arrays develop and mature; we have attempted to indicate the spatial sampling coverage likely over the next 5 years. Note that the mooring box includes both open-ocean observatories and compact, fixed observatories deployed in coastal and benthic regions. Box boundaries that are directly adjacent to one another (i.e., the upper boundaries of Profiling floats, Decadal Hydrographic Survey, and Volunteer Observing Ships) indicate the same temporal or spatial boundary but are offset for clarity

decades, making them suitable to observe variability and long-term changes in ocean chemistry at a given location (Fig. 1). Moorings are a relatively large-capacity platform that can be serviced regularly, allowing for the deployment of complex systems with minimal size and energy constraints. The most common and widespread carbonate system observations on moorings are of $p \mathrm{CO}_{2}$ by the Moored Autonomous $p \mathrm{CO}_{2}\left(\mathrm{MAPCO}_{2}\right)$ system [33]. Originally developed from the technology used on shipboard underway systems [34], the $\mathrm{MAPCO}_{2}$ system sequentially measures atmospheric and seawater $p \mathrm{CO}_{2}$ using a nondispersive infrared detector. These measurements yield the $p \mathrm{CO}_{2}$ difference between air and water $\left(\Delta p \mathrm{CO}_{2}\right)$ with an uncertainty of $\pm 2 \mu \mathrm{atm}$ [33]. A key characteristic of this system is the ability to conduct frequent 
in situ calibrations using traceable standard gases to achieve climate-quality $p \mathrm{CO}_{2}$ data when combined with careful preand post-deployment quality control and calibration [33].

$\mathrm{MAPCO}_{2}$ systems are deployed in a global network at over 40 locations, ranging from open ocean sites to the coastal ocean, with the oldest records spanning up to 15 years (www.pmel.noaa.gov/co2/, [35, 36]). These moorings record year-round observations at most sites, characterizing subseasonal to interannual, as well as regional variability of $p \mathrm{CO}_{2}$ in an array that can be used to understand the largescale processes that influence air-sea carbon dioxide fluxes. For example, long-term observations in a tropical coral reef system demonstrated the importance of various processes that influence air-sea $\mathrm{CO}_{2}$ flux including rainfall, nutrient delivery, winds, and local biological communities [37, 38]. Sustained mooring observations in the Tropical Pacific revealed that climate forcings such as El Niño/La Niña cycles [35, 39] and anomalous warming events in the Pacific [40] are dominant drivers for interannual variability of $p \mathrm{CO}_{2}$. Furthermore, these surface observations of $p \mathrm{CO}_{2}$ are compiled along with shipboard observations into the Surface Ocean $\mathrm{CO}_{2}$ Atlas (SOCAT) [24], providing crucial information about the seasonal cycle for calculating global air-sea $\mathrm{CO}_{2}$ fluxes (e.g., [41, 42]).

In addition to using surface observations of $\mathrm{CO}_{2}$ to quantify large-scale air-sea fluxes and understand the ocean's role in the global carbon cycle, surface $\mathrm{CO}_{2}$ observations have led to an increased understanding of biogeochemical processes in the upper ocean. In order to understand biogeochemical processes, a second carbonate parameter is needed to fully constrain the carbonate system. $\mathrm{pH}$ sensors have been developed for moorings (e.g., [43]) but the strong covariance of $p \mathrm{CO}_{2}$ and $\mathrm{pH}$ means that uncertainties in either measurement translate to large uncertainties in the other calculated carbonate system parameters that greatly exceed the uncertainty from using two parameters that covary less strongly [19, 44, 45]. Empirical algorithms relating TA to commonly measured surface variables have allowed the use of single carbonate system parameters such as $p \mathrm{CO}_{2}$ to yield new understanding of surface carbonate chemistry. For example, in a pair of papers, Fassbender et al. [19, 20] used mooring observations from Ocean Station Papa (Gulf of Alaska) and the Kuroshio Extension to decompose surface biological production into its organic and inorganic components. Similarly, the equatorial $p \mathrm{CO}_{2}$ observations used to characterize the relationship between $p \mathrm{CO}_{2}$ and $\mathrm{El} \mathrm{Niño/La} \mathrm{Niña} \mathrm{were} \mathrm{combined} \mathrm{with}$ an algorithm estimate for TA to determine that $\mathrm{pH}$ in the region was more variable and changing faster than expected [35]. In coastal regions, $\mathrm{MAPCO}_{2}$ measurements off the Eastern coast of the United States were used to show that riverine input and local biological production and respiration were strong drivers of seasonal cycles in $p \mathrm{CO}_{2}$ [46], while annual coral reef calcification rates were estimated for a rim reef near Bermuda [47].
While the $\mathrm{MAPCO}_{2}$ network is providing highly accurate observations and playing a critical role in our understanding of the global ocean carbonate cycle, there are some limitations. For example, moorings cannot provide spatial context to the temporal variability they observe. Furthermore, the costs associated with maintaining and servicing the moorings, especially in the open ocean, make it unlikely that we will be able to significantly increase the size of the mooring array. Finally, subsurface processes greatly affect surface carbonate chemistry; thus, while surface variability can be observed from $\mathrm{MAPCO}_{2}$ sensors on moorings, the technology employed on these buoys requires frequent in situ calibration using standard gases, which makes it unsuitable for subsurface measurements.

\section{Compact, Fixed Observatories}

In recent years, a number of in situ sensors for carbonate chemistry have become commercially available, making autonomous measurements more accessible to the community [31]. These sensors allow for routine deployments in a wide range of ecosystems by research groups that are not necessarily experts in instrumentation. We make a distinction from the surface mooring $p \mathrm{CO}_{2}$ systems in the previous section, as these sensors are smaller (i.e., can be carried by a single person), can make subsurface measurements, and do not require a large surface mooring for deployment.

$\mathrm{pH}$ and $p \mathrm{CO}_{2}$ sensors are available from multiple vendors that utilize a range of sensing techniques [31]. The International Ocean Carbon Coordination Project (www. ioccp.org) maintains an online database with the current status of technology for carbonate chemistry instrumentation. The development of $\mathrm{pH}$ sensors based on the Honeywell DuraFET Ion Sensitive Field Effect Transistor (ISFET) technology is arguably one of the most significant recent advancements in autonomous carbonate chemistry measurements. The DuraFET was originally designed for industrial applications [48], but was adapted for oceanographic use after demonstrating excellent stability and performance in seawater [49]. Nernstian behavior over large ranges in $\mathrm{pH}$ and salinity was observed, allowing for accurate measurements of $\mathrm{pH}$ [50]. The DuraFET was first adapted for shallow water applications [51], and was further modified for high-pressure, profiling float applications [52]. The details and applications for the latter will be presented in the next section. In this section, we highlight studies that utilized self-contained autonomous sensors in fixed locations to investigate carbonate dynamics, with an emphasis on coastal systems.

Nearshore coastal ecosystems are among the most productive in the world and are significant contributors to biodiversity [53]. They also provide huge societal benefits through storm protection and water quality improvement, and provide billions of dollars in revenue from fishing, recreation, and 
tourism [54]. Many organisms that are thought to be vulnerable to ocean acidification reside in these habitats, such as bivalves, corals, and calcifying algae [10]. Coastal ecosystems are highly dynamic, and can experience natural variability on timescales ranging from hours to interannual (Fig. 1). The magnitude of this natural variability can in some cases be significantly larger than the expected changes due to ocean acidification [55]. High biomass and productivity, a shallower water column, and more pronounced changes in physical conditions contribute to high-frequency variability, which is difficult or impossible to capture using discrete sampling approaches [56].

Compact fixed observatories are particularly useful in characterizing high-frequency variability, and helped guide ocean acidification research in investigating the impacts of natural variability. For example, Hofmann et al. [55] compiled $\mathrm{pH}$ sensor data from ecosystems ranging from Antarctica to tropical coral reefs, and presented distinct biome-specific $\mathrm{pH}$ patterns that occur on diel, semi-diel, and stochastic timescales. This led to further investigation into exploring patterns of variability in ecosystems such as kelp forests [57, 58], coral reefs [59-62], seagrass meadows [63, 64], continental shelf [65], and upwelling regions [66, 67]. Diel $\mathrm{pH}$ variability in coral reefs was found to correlate with community structure and net accretion rates [68], suggesting the potential for natural variability to influence impacts of ocean acidification. Such observations are driving studies to examine the role natural variability plays in organismal response in ocean acidification experiments [69-72].

High-resolution coastal data can also be used to model future carbonate conditions using habitat-specific ocean acidification models. For example, the first high-frequency nearshore record of $\mathrm{pH}$ from under sea ice in Antarctica was used to model future wintertime $\mathrm{pH}$ [73]. Takeshita et al. [67] decomposed $\mathrm{CO}_{2}$ variability into its natural and anthropogenic components and used different atmospheric $\mathrm{CO}_{2}$ pathways to model future conditions over an upwelling shelf. The full carbonate system was reconstructed and projected to the end of the century by combining sensor time series and a mechanistic model for a seagrass bed [64]. Development of autonomous systems that can directly measure key fluxes such as benthic metabolism [74, 75] or air-sea flux [76] will help in properly parameterizing such models [77]. These model outputs can act as another guide for experimental conditions in ocean acidification studies of ecosystem responses, a crucial complement to large-scale open-ocean observing systems.

\section{Global Ocean Observations from Profiling Floats}

Profiling floats are the only autonomous observational platform that has been demonstrated to be scalable to a global level for any measurement, and are particularly suited to study basin-wide to global processes on seasonal to interannual timescales (Fig. 1). The Argo profiling float array currently consists of approximately 4000 floats, returning temperature and salinity profiles from $2000 \mathrm{~m}$ to the surface every 10 days from around the globe [26]. Given the ability of a float array to make observations on seasonal to interannual timescales at basin to global scales, significant effort has been devoted to integrating biogeochemical sensors onto profiling floats, including oxygen [78, 79], nitrate [80], and bio-optical measurements for chlorophyll $a$ fluorescence [81] and particle backscatter [82]. These biogeochemical profiling floats have been used to study production dynamics [83-87], nutrient delivery to oligotrophic waters [88], oxygen minimum zone dynamics [89], regional air-sea fluxes [90-92], and elemental ratios [93]. $\mathrm{A} p \mathrm{CO}_{2}$ sensor has been integrated onto a profiling float [94], but has not left the prototyping phase due to issues such as long response time, need for frequent recalibration, and high power requirements. An ISFET-based $\mathrm{pH}$ sensor represents the most recent addition to the sensor suite available for biogeochemical profiling floats and has been demonstrated to be robust and stable throughout the depth range and lifetime of floats [52].

Until recently, most biogeochemical profiling floats were deployed in small numbers by individual researchers or small groups. Building on the success of these individual programs, the Southern Ocean Carbon and Climate Observations and Modeling (SOCCOM) program began the first attempt at creating a biogeochemical float array at the basin scale [95]. The Southern Ocean plays a disproportionate role in moderating the climate through heat uptake, anthropogenic $\mathrm{CO}_{2}$ uptake [96], and nutrient delivery to the thermocline [97], yet remains chronically under-sampled due to its remoteness and harsh conditions, especially during the Austral winter. To address this, the SOCCOM project began deploying biogeochemical profiling floats in 2014 with the goal of establishing an array of 200 floats over 6 years [95]. The floats are equipped with oxygen, nitrate, $\mathrm{pH}$, and bio-optical sensors, and provide measurements every 10 days. Currently, there are over $120 \mathrm{pH}-$ equipped floats operating in the Southern Ocean (soccom. princeton.edu), demonstrating that technological challenges have been overcome to operate large biogeochemical float arrays. After post-deployment quality control, (described in the "Challenges" section), these float $\mathrm{pH}$ measurements show excellent agreement with independent bottle samples collected at the time of deployment to $+0.005 \pm 0.01(n=952$ bottle samples; updated from Johnson et al. [95]).

The array of biogeochemical floats in the Southern Ocean is providing novel insights into air-sea $\mathrm{CO}_{2}$ fluxes and biogeochemical processes. For example, large discrepancies in winter surface $p \mathrm{CO}_{2}$ between float observations and climatologies based on shipboard observations [25] are consistently observed [98-101]. This is not particularly surprising because wintertime shipboard data are sparse. However, the implications for this discrepancy are potentially immense. An initial 
analysis of 35 floats over 3 years in the Southern Ocean found an annual uptake of only $0.08 \pm 0.55 \mathrm{Pg} \mathrm{C}_{\text {year }}{ }^{-1}$, instead of the $\sim 1 \mathrm{Pg} \mathrm{C}$ year $^{-1}$ uptake calculated from ship-based estimates, due primarily to increased wintertime outgassing around the Polar Front [100]. This discrepancy represents approximately $50 \%$ of the annual contemporary global oceanic $\mathrm{CO}_{2}$ uptake [1], and would pose a major challenge to our understanding of the global carbon budget by effectively eliminating the role of the Southern Ocean as a carbon sink. However, a follow-up study combining the float observations with the mooring and underway $p \mathrm{CO}_{2}$ dataset (SOCAT) indicates that while the outgassing observed by the SOCCOM floats is real and significant, it likely represents a more modest reduction in the Southern Ocean carbon uptake (Bushinsky et al., in review, Global Biogeochemical Cycles).

Float-based estimates of other carbonate system parameters can be used in conjunction with biogeochemical sensor data such as nitrate or oxygen as an additional constraint on biogeochemical processes. Williams et al. [99] combined SOCCOM float-derived carbonate system estimates and nitrate data to decompose the seasonal drivers of the carbonate system, finding that carbonate system seasonal cycles agree well with previous climatologies in the spring and summer months but differ in winter months when data were previously sparse. Measurements of biogeochemical parameters under sea ice have been particularly lacking, and this is especially true for carbonate observations. Estimated DIC from underice floats was used to help quantify under-ice heterotrophy, but yielded intriguing stoichiometric ratios between inorganic carbon, oxygen, and nitrate that warrant more exploration [102]. These studies highlight the large knowledge gaps that could only be revealed through an array of sustained, autonomous observations.

\section{Emerging Technologies}

In this section, we discuss the likely platforms and sensors that will comprise our near- and long-term autonomous future in observing the carbonate system. One notable development currently underway is the transition of relatively mature carbonate observing sensors from moorings and floats to gliders and autonomous surface vehicles, which involves repackaging of systems rather than development of new sensors. We also discuss new sensing technology for carbonate sensors and the opportunities they might bring.

While moorings and an ever-increasing number of profiling floats are sampling the open ocean and small-scale local observatories have been deployed in many near-shore locations, there is currently a gap in autonomous carbonate observations of regional processes. Coastal regions, boundary currents, and other meso- and submesoscale processes all have significant importance to the global carbon cycle but currently lack sustained autonomous observations for carbonate chemistry [103]. These regions have higher spatial and temporal variability than can easily be sampled by ships and are either too shallow or have fast moving currents that limit sampling by profiling floats. For example, the California coastal upwelling region is both an important fishery and likely to experience early effects of acidification. Cruise transects have observed significant seasonal upwelling-driven corrosive waters $(\Omega<1)$ on the shelf [104] and some areas experience persistent undersaturated conditions [105]. Coastal glider transects using buoyancy-driven vehicles in the California Current System, as part of the California Cooperative Oceanic Fisheries Investigations (CalCOFI) have been used to calculate $\Omega$ [106] using empirical relationships with temperature and oxygen [107], finding periodic undersaturation in nearshore waters.

Underwater gliders have been demonstrated to be an effective platform at studying submeso- to mesoscale processes such as fronts and eddies, and to connect the coastal ocean to the open ocean [27]. Equipping gliders with carbonate parameter sensors should provide a much more spatially and temporally detailed understanding in these complex and important regions. Developing new, or adopting existing sensing technology for mobile profiling platforms is challenging, as there are significant constraints on size, reagent consumption, power, response time, and requires well-characterized dynamic errors as the platform moves through the water column. Despite these challenges, there are some larger powered autonomous underwater vehicles (AUVs) that have been equipped with $p \mathrm{CO}_{2}$ sensors [108] and initial test deployments of $\mathrm{pH}$ on gliders look promising $[109,110]$. Such regions will also likely require integration of overlapping vehicles and platforms to make best use of the tradeoffs in duration, capacity, and sensor capabilities of our available observing capabilities (Fig. 1).

Another powerful platform for carbonate observations are autonomous surface vehicles powered by wind or waves. Vehicles such as Wave Gliders [111] and Saildrones [112] are faster and more mobile than buoyancy-driven gliders but more expensive to operate and typically have shorter deployment durations. $\mathrm{A} \mathrm{CO}_{2}$ system originally designed for mooring operations [34] and an ISFET $\mathrm{pH}$ sensor mounted on a Wave Glider have produced high-resolution observations of surface $p \mathrm{CO}_{2}$ and calculated air-sea $\mathrm{CO}_{2}$ fluxes in Monterey Bay, California [113]. Similarly, Saildrones have been equipped with modified $\mathrm{MAPCO}_{2}$ systems [114] and DuraFET $\mathrm{pH}$ sensors and are capable of sampling fast moving currents that may be inaccessible to profiling floats or gliders.

The development and refinement of existing sensing technology will be essential to expanding our autonomous observing capabilities. For example, refinement of sensor design and conditioning significantly reduced initial drift for $\mathrm{pH}$ sensors 
on profiling floats [95]. Continuing to improve our current sensor designs for reliability, performance, and reduction in cost is an underappreciated yet important task. Solid state, low power optical sensors for $\mathrm{pH}$ and $p \mathrm{CO}_{2}$ that use similar sensing principles to the successful and widespread $\mathrm{O}_{2}$ sensor have shown promising results in the laboratory and on autonomous platforms $[115,116]$. The development of robust, autonomous measurements for additional parameters such as DIC, TA, or carbonate ion concentration $\left(\left[\mathrm{CO}_{3}{ }^{2-}\right]\right)$ is also highly desired. While using empirically estimated TA is appropriate for some situations, this approach will not work in many coastal systems such as estuaries and coral reefs. Prototype in situ measurement systems for DIC [45, 117-119] and TA [120] have been successfully deployed with encouraging results. However, all of these approaches utilize multiple pumps and valves, making power consumption, size, robustness, and reliability major challenges to overcome. Development of "labon-chip" devices has made great strides toward conducting in situ chemistry on microfluidic scales [121], and could provide a path forward for such in situ analyzers. Successful deployments of lab-on-chip devices on underwater gliders have been demonstrated for nitrate [153]. Innovative TA sensors that generate hydrogen ions in situ through coulometry [122] or ionselective membranes [123] to conduct chronopotentiometric titrations have also been demonstrated in the laboratory. These sensors are small, solid state, and low power, making them promising candidates for in situ sensing applications.

Recently, a method for accurate $\left[\mathrm{CO}_{3}{ }^{2-}\right]$ measurement based on spectrophotometry has been developed [124-126]. $\left[\mathrm{CO}_{3}{ }^{2-}\right]$ measurements are particularly useful when the primary target is saturation state. The measurement principle is very similar to spectrophotometric $\mathrm{pH}$, and thus should be adaptable for autonomous applications. $\left[\mathrm{CO}_{3}{ }^{2-}\right]$ is used to calculate the saturation state of calcium carbonate $(\Omega)$ and the ability to measure $\left[\mathrm{CO}_{3}{ }^{2-}\right]$ adds a fifth "master" variable to evaluate the carbonate system $[4,125]$. This measurement could become a new and exciting tool to monitor ocean acidification [127, 128], especially in coastal areas where low saturation state is thought to be the primary driver for deleterious impacts from ocean acidification for organisms such as bivalve larvae [13, 129] and pteropods [130].

The amount of particulate carbon produced in the form of calcium carbonate relative to primary production of organic carbon is an important but poorly constrained component of the carbonate cycle [131]. Profiling floats have been equipped with optical sediment traps to estimate vertical particle fluxes out of the upper ocean. Initial results indicate these can be converted into carbon flux estimates [132]. The highly specialized Carbon Flux Explorer profiling floats can observe sinking particles and determine particulate inorganic carbon rain rates in addition to organic carbon, finding significantly greater export in the wintertime than indicated by more basic sediment traps [133]. These technologies may provide an additional constraint on estimates of inorganic carbon production from measured carbonate system parameters.

\section{Challenges}

In addition to the development of new sensing technology, similar amounts of effort should be devoted to the development, adoption, and validation of robust calibration protocols. Calibration protocols are essential for successfully operating a network of autonomous platforms, as they ensure accuracy and consistency throughout the array, and sensor drift can be identified and corrected. Such a protocol has been established and implemented for $\mathrm{pH}$ on profiling floats. The conductivity sensor (used to calculate salinity) on Argo floats is corrected using deep waters ( $>1500 \mathrm{~m}$ ), as conditions are stable and can be predicted using hydrographic measurements [134]. Following this approach, $\mathrm{pH}$ sensors are corrected by comparing sensor $\mathrm{pH}$ to a deep reference $\mathrm{pH}$ field at $1500 \mathrm{~m}$ [95]. The reference $\mathrm{pH}$ field is calculated from empirical relationships derived from hydrographic data using temperature, salinity, pressure, and oxygen as inputs [135]. These algorithms can be region-specific [135] or global [136-138]. It should be noted that the accuracy of the reference $\mathrm{pH}$ field, and thus corrected sensor $\mathrm{pH}$, depends on other parameters measured on the float such as temperature, salinity, pressure, and oxygen [98]. Therefore, the quality of calibration for the other sensors will affect the final corrected $\mathrm{pH}$ data as well. Furthermore, regions where anthropogenic carbon has penetrated into the deep ocean such as the North Atlantic will require regular updates to the algorithms, or inclusion of more robust temporal trends in the algorithm [136]. The quality of float $\mathrm{pH}$ data is similar to those from state-of-the-art hydrographic cruises, demonstrating the capability of accurately observing carbonate chemistry from autonomous profiling arrays.

Validation of the SOCCOM calibration protocols has been achieved through the hydrographic casts and discrete bottle data that typically accompany each float deployment. While this has been instrumental for the development and validation of quality control protocols [95], it is not feasible to accompany every biogeochemical float deployment with a full cast of discrete bottle samples. Thus, it will be important to prepare for deployment strategies without validation samples, while continuing to assess the performance of this quality control protocol in different ocean basins. Furthermore, data adjustment based on deep reference $\mathrm{pH}$ fields is not always possible, such as for moorings, shallow water process studies, or smallscale observatories. Integrating in situ calibration functionality using certified reference materials such as tris buffer for $\mathrm{pH}$ [139] will allow for consistent and accurate datasets across multiple platforms and research groups. As more sensing technologies continue to become available, establishment of best 
practices [51] to ensure proper calibration, quality control, and sensor intercomparability will be essential.

In addition to calibration of individual carbonate sensors, it is essential that the uncertainties in the estimated carbonate variables are critically assessed. For example, a careful bottom-up uncertainty analysis yielded an estimate of \pm $2.86 \%$ uncertainty in the SOCCOM float-based $p \mathrm{CO}_{2}$ estimates, equating to approximately $\pm 11.4 \mu \mathrm{atm}$ at $400 \mu \mathrm{atm}$ $p \mathrm{CO}_{2}$ [98]. This represents the absolute accuracy of a single float, but the ability of $\mathrm{pH}$-derived $\mathrm{pCO}_{2}$ to track spatiotemporal patterns over, e.g., a seasonal cycle is significantly better and is estimated to be $\pm 5.5 \mu \mathrm{atm}$ [140]. However, the seasonal to multi-annual long-term stability of surface $p \mathrm{CO}_{2}$ estimates has not been characterized. No studies have investigated in equal detail possible biases in estimates of DIC or $\Omega$. Furthermore, it is important to distinguish between systematic biases that affect the entire array in the same direction and random uncertainties in individual observations or sensors that are uncorrelated and will average out over the fleet. Small systematic biases in estimated $p \mathrm{CO}_{2}$ can have a large impact when scaled up to global air-sea flux estimates. The average difference between shipboard underway $p \mathrm{CO}_{2}$ and float-estimated $p \mathrm{CO}_{2}$ is $3.7 \mu \mathrm{atm} p \mathrm{CO}_{2}(n=35-39)$ [99, 100]. However, it is unclear whether or not this represents a real systematic bias in the float estimates, or an artifact due to small sample size and spatiotemporal variability $( \pm 1$ day and $\pm 25 \mathrm{~km}$ ) between float and ship measurements. Increased efforts to validate float $p \mathrm{CO}_{2}$ estimates and determine the magnitude of any bias are crucial as these biogeochemical Argo float deployments expand.

Several issues have been identified in our current thermodynamic model of the marine carbonate system. A recent analysis demonstrated that uncertainty in carbonate system equilibrium constants was a dominant source of error when other parameters in the carbonate system were calculated [141]. For example, a pH-dependent bias between directly measured $\mathrm{pH}$ using spectrophotometry and $\mathrm{pH}$ calculated from TA and DIC has been identified [136], though the scope and nature of the bias is still under investigation by the community. This has implications for how to calibrate $\mathrm{pH}$ sensors using deep values, and its associated uncertainty when estimating surface $p \mathrm{CO}_{2}$ [98]. These thermodynamic parameters are characterized through careful laboratory experiments and are not as well defined for some of the less commonly sampled waters such as in sea ice regions and coastal waters. In the case of sea ice, where waters are routinely below $0{ }^{\circ} \mathrm{C}$, commonly used carbonate system constants do converge, but brackish coastal waters may require more work [142, 143]. Coastal waters may also contain an unknown contribution of organic alkalinity that is not parameterized in the inorganic carbonate model [144, 145]. These results have highlighted the need for more research into understanding where the uncertainties in the carbonate system may lie [146].
As introduced earlier, float $\mathrm{pH}$ measurements can be combined with empirical algorithms of TA to calculate other carbonate parameters. Recently, two new algorithms have been developed to estimate TA and other carbonate system parameters on a global scale based on the same global ocean discrete bottle sample dataset [29]. The LIR (Locally Interpolated Regression) uses a multiple linear regression approach, and interpolates the coefficients of the regression model to any location [136, 147], providing a smooth transition between region-specific relationships. The second approach is CANYON (CArbonate system and Nutrients concentration from hYdrological properties and Oxygen using a Neural-network), which uses a neural network [137, 138]. Both approaches are capable of estimating TA with uncertainties of about $\pm 6-8 \mu \mathrm{mol} \mathrm{kg}^{-1}$ globally, though a detailed comparison between these two algorithms has not been performed and region-specific algorithms may still be necessary [98]. These global algorithms are an enabling step for a global profiling float array and other autonomous platforms as they provide a framework to calibrate future sensors as the array continues to expand.

Until widespread observations of multiple carbonate parameters are possible, the community will likely continue to rely on algorithm and mapping approaches for carbonate system parameters. Simultaneously measuring two carbonate parameters would alleviate the need to rely on algorithm approaches to estimate TA, but would not remove the need to interpolate and extrapolate from available observations to provide spatially resolved maps. While improvements in mapping methods (e.g., $[41,138])$ are an essential component in understanding the oceanic $\mathrm{CO}_{2}$ flux on annual to decadal timescales, these products still suffer from any biases that exist in the underlying observational dataset and will require the ongoing addition of in situ data to maintain their utility. Just as new profiling float-based estimates of $p \mathrm{CO}_{2}$ indicate that the interpolation schemes used for surface observations of $p \mathrm{CO}_{2}$ cannot reproduce a signal they do not observe, it is likely that issues will arise with the use of empirical algorithms for TA and other carbonate system parameters. For example, in regions with highly variable $\mathrm{CaCO}_{3}$ production, empirical algorithms based on temperature, salinity, and oxygen alone will not necessarily capture changes in TA associated with calcification and dissolution. Additionally, these algorithms must be continually updated with new training data as surface conditions change due to acidification and natural interannual or decadal variability. Therefore, high-quality ship-based observational programs such as the repeat hydrography and time series programs are critical and will remain a fundamental component of our autonomous future.

The expansion of autonomous observations of the carbonate system should be matched with an increased ability to use and interpret these new measurements. While the makeup of our developing observing system is still in flux, it is clear that 
the network will be a mix of stationary and mobile platforms that produce both direct measurements and derived quantities. Integration of these autonomous observations with conventional shipboard measurements for use in data synthesis and modeling approaches will be key to leveraging our existing and future capabilities for maximum benefit.

Continued advancement of data management is required to deal with the increasing volume and types of data to make it accessible to the outside community, especially modelers. These data must be readily available, well-documented, and in a user-friendly format to be useful. For example, providing data in a unified data format and stored in central locations has been crucial to the success of the core Argo array, but addition of biogeochemical parameters presents new challenges in post-deployment quality control and data management. The development of cyber infrastructure to deal with differing data formats, recording and propagation of uncertainties, and making data available for near-real-time assimilation into forecast models will all make these datasets more useful. Coordination between the observation, modeling, and data management communities from the early stages of planning will help with this effort.

A successful example of such coordination between communities has been demonstrated by the SOCCOM project [148]. During the planning stages of the project, Observing System Simulation Experiments (OSSEs) were used to simulate an array of Southern Ocean biogeochemical profiling floats and to examine the reconstruction skill that could be achieved from the fleet $[149,150]$. These OSSEs provided critical information about the number of floats required to reproduce observed patterns of oxygen, DIC, and air-sea $\mathrm{CO}_{2}$ fluxes. The development of the Biogeochemical Southern Ocean State Estimate (B-SOSE; [151]), a data-assimilating numerical model which seeks to minimize model-observation differences using conserved model dynamics, now provides observationally informed output in a gridded format useful for prognostic model evaluation [152]. Looking ahead, the use of observations in conjunction with models, either through assimilating state estimate models or to validate prognostic models, is a powerful tool for synthesizing existing observations and extrapolating our understanding into the future.

\section{Conclusions}

The past 5 years have seen the expansion of our autonomous carbonate observing system. Existing observations by moorings have become an integral part of the community's ability to estimate large-scale $\mathrm{CO}_{2}$ fluxes which are critical to our understanding of the ocean's role in climate. Float-based estimates of $p \mathrm{CO}_{2}$ are supplementing these observations, adding new information about previously poorly sampled seasonal cycles. The impact of new pH sensors deployed on SOCCOM biogeochemical profiling floats has demonstrated how basinscale measurements of the carbonate system can fundamentally alter our understanding of the carbon cycle. Expansion of this approach globally could be an invaluable addition to our carbonate observing system, complementing high-accuracy shipboard observations and broadening our understanding of the global carbonate system. These large-scale observations have been matched by the increase in small-scale observatories in coastal shelf ecosystems and coral reefs.

While our ability to observe the carbonate system has expanded dramatically in recent years, we are often limited to observations of only one carbonate system parameter at a time. For now, empirical estimates of total alkalinity have provided sufficient information to calculate the entire carbonate system, greatly enhancing the value of existing observations. Looking forward, there are many new sensors in the pipeline that will either enable observation on more autonomous platforms or observation of different carbonate system parameters. Expansion of mature sensors onto glider platforms and fast, mobile surface platforms are especially exciting as the integration of sensors and vehicles is already underway. We are currently in the midst of a massive change in observational capability for the ocean biogeochemical community and close to a future where the most important processes impacting the ocean carbonate system will be observable at high accuracy and at high-resolution spatial and temporal scales.

Acknowledgments SMB is supported by the Southern Ocean Carbon and Climate Observations and Modeling (SOCCOM) Project, funded by the National Science Foundation, Division of Polar Programs (NSF PLR -1425989), NASA (NNX17AI73G), and the Carbon Mitigation Initiative (CMI) project sponsored by BP at Princeton University. YT was supported by David and Lucile Packard Foundation. NLW was supported by the National Academies of Sciences through the Research Associateship Programs Postdoctoral Fellowship. This is PMEL contribution number 4924. We would also like to thank Ken Johnson, Jorge Sarmiento, and Richard Feely for comments on this manuscript. We thank Sarah Battle for her excellent assistance in making the figure that accompanies this manuscript. Finally, we thank two anonymous reviewers for their helpful feedback.

\section{Compliance with Ethical Standards}

Conflict of Interest On behalf of all authors, the corresponding author states that there is no conflict of interest.

Human and Animal Rights This article does not contain any studies with human or animal subjects performed by any of the authors.

Open Access This article is distributed under the terms of the Creative Commons Attribution 4.0 International License (http:// creativecommons.org/licenses/by/4.0/), which permits unrestricted use, distribution, and reproduction in any medium, provided you give appropriate credit to the original author(s) and the source, provide a link to the Creative Commons license, and indicate if changes were made. 


\section{References}

1. Le Quéré C, Andrew RM, Friedlingstein P, Sitch S, Hauck J, Pongratz J, et al. Global Carbon Budget 2018. Earth Syst Sci Data. 2018;10:2141-94.

2. Sabine CL, Tanhua T. Estimation of anthropogenic $\mathrm{CO}_{2}$ inventories in the ocean. Annu Rev Mar Sci. 2010;2:175-98.

3. Canadell JG, Le Quere C, Raupach MR, Field CB, Buitenhuis ET, Ciais $\mathrm{P}$, et al. Contributions to accelerating atmospheric $\mathrm{CO} 2$ growth from economic activity, carbon intensity, and efficiency of natural sinks. Proc Natl Acad Sci. 2007;104:18866-70.

4. Zeebe RE. History of seawater carbonate chemistry, atmospheric $\mathrm{CO}_{2}$, and ocean acidification. Annu Rev Earth Planet Sci. 2012;40:141-65.

5. Millero FJ. The marine inorganic carbon cycle. Chem Rev. 2007; 107:308-41.

6. Feely RA, Sabine CL, Lee K, Berelson W, Kleypas J, Fabry VJ, et al. Impact of anthropogenic $\mathrm{CO}_{2}$ on the $\mathrm{CaCO}_{3}$ system in the oceans. Science. 2004;305:362-6.

7. Bates N, Astor Y, Church M, Currie K, Dore J, Gonaález-Dávila $\mathrm{M}$, et al. A time-series view of changing ocean chemistry due to ocean uptake of anthropogenic $\mathrm{CO}_{2}$ and ocean acidification. Oceanography. 2014;27:126-41

8. Honisch B, Ridgwell A, Schmidt DN, Thomas E, Gibbs SJ, Sluijs A, et al. The geological record of ocean acidification. Science. 2012;335:1058-63.

9. Doney SC, Fabry VJ, Feely RA, Kleypas JA. Ocean acidification: the other CO2 problem. Annu Rev Mar Sci. 2009;1:169-92.

10. Kroeker KJ, Kordas RL, Crim R, Hendriks IE, Ramajo L, Singh GS, et al. Impacts of ocean acidification on marine organisms: quantifying sensitivities and interaction with warming. Glob Chang Biol. 2013;19:1884-96.

11. Fabry VJ, Seibel BA, Feely RA, Orr JC. Impacts of ocean acidification on marine fauna and ecosystem processes. Int Counc Explor Sea. 2008;65:414-32.

12. Bednaršek N, Feely RA, Tolimieri N, Hermann AJ, Siedlecki SA, Waldbusser GG, et al. Exposure history determines pteropod vulnerability to ocean acidification along the US West Coast. Sci Rep. 2017;7:1-12.

13. Barton A, Waldbusser GG, Feely RA, Weisberg SB, Newton JA, Hales B, et al. Pacific Northwest shellfish industry. Oceanography. 2015;28:146-59.

14. Albright R, Takeshita Y, Koweek DA, Ninokawa A, Wolfe K, Rivlin T, et al. Carbon dioxide addition to coral reef waters suppresses net community calcification. Nature. 2018;555:516-9.

15. Gruber N, Hauri C, Lachkar Z, Loher D, Frölicher TL, Plattner G$\mathrm{K}$. Rapid progression of ocean acidification in the California current system. Science. 2012;337:220-3.

16. Steinacher M, Joos F, Frölicher TL, Plattner G-K, Doney SC. Imminent ocean acidification in the Arctic projected with the NCAR global coupled carbon cycle-climate model. Biogeosciences. 2009;6:515-33.

17. Hauri C, Friedrich T, Timmermann A. Abrupt onset and prolongation of aragonite undersaturation events in the Southern Ocean. Nat Clim Chang. 2016;6:172-6.

18. Wanninkhof R. Relationship between wind speed and gas exchange. J Geophys Res. 1992;97:7373-82.

19. Fassbender AJ, Sabine CL, Cronin MF. Net community production and calcification from 7 years of NOAA Station Papa Mooring measurements. Glob Biogeochem Cycles. 2016;30: 250-67.

20. Fassbender AJ, Sabine CL, Cronin MF, Sutton AJ. Mixed layer carbon cycling at the Kuroshio Extension Observatory. Glob Biogeochem Cycles. 2017:1-17.
21. Sabine CL, Feely RA, Gruber N, Key Robert M, Lee K, Bullister John L, et al. The oceanic sink for anthropogenic CO2. Science. 2004;305:367-71.

22. Talley LD, Feely RA, Sloyan BM, Wanninkhof R, Baringer MO, Bullister JL, et al. Changes in ocean heat, carbon content, and ventilation: a review of the first decade of GO-SHIP Global Repeat Hydrography. Ann Rev Mar Sci. 2016;8:annurev-marine052915-100829.

23. Gruber N, Clement D, Carter BR, Feely RA, van Heuven S, Hoppema M, et al. The oceanic sink for anthropogenic CO 2 from 1994 to 2007. Science. 2019;363:1193-9.

24. Bakker DCE, Pfeil B, Landa CS, Metzl N, O'Brien KM, Olsen A, et al. A multi-decade record of high-quality $\mathrm{fCO}_{2}$ data in version 3 of the Surface Ocean $\mathrm{CO}_{2}$ Atlas (SOCAT). Earth Syst Sci Data. 2016;8:383-413.

25. Takahashi T, Sutherland SC, Wanninkhof R, Sweeney C, Feely RA, Chipman DW, et al. Climatological mean and decadal change in surface ocean $\mathrm{pCO}_{2}$, and net sea-air $\mathrm{CO}_{2}$ flux over the global oceans. Deep Res Part II Top Stud Oceanogr. 2009;56:554-77.

26. Riser SC, Freeland HJ, Roemmich D, Wijffels S, Troisi A, Belbéoch M, et al. Fifteen years of ocean observations with the global Argo array. Nat Clim Chang Nature Publishing Group. 2016;6:145-53.

27. Rudnick DL. Ocean research enabled by underwater gliders. Annu Rev Mar Sci. 2016;8:519-41.

28. Hobson BW, Bellingham JG, Kieft B, McEwen R, Godin M, Zhang YW. 2012. Tethys-class long range AUVs-extending the endurance of propeller-driven cruising AUVs from days to weeks. In 2012 IEEE/OES Autonomous Underwater Vehicles (AUV): Southampton, UK, September 24-27, 2012. New York: IEEE. http://ieeexplore.ieee.org/xpl/ articleDetails.jsp?arnumber $=6380735$

29. Olsen A, Key RM, Van Heuven S, Lauvset SK, Velo A, Lin X, et al. The global ocean data analysis project version 2 (GLODAPv2) - an internally consistent data product for the world ocean. Earth Syst Sci Data. 2016;8:297-323.

30. Carter BR, Williams NL, Evans W, Fassbender AJ, Barbero L, Hauri C, Feely RA, Sutton AJ Time-of-detection as a metric for prioritizing between climate observation quality, frequency, and duration. Geophys Res Lett 2019;46:3853-3861.

31. Martz T, Daly K, Byrne R, Stillman J, Turk D. Technology for Ocean Acidification Research: needs and availability. Oceanography. 2015;25:40-7.

32. Byrne RH. Measuring ocean acidification: new technology for a new era of ocean chemistry. Environ Sci Technol. 2014;48:5352-60.

33. Sutton AJ, Sabine CL, Maenner-Jones S, Lawrence-Slavas N, Meinig C, Feely RA, et al. A high-frequency atmospheric and seawater $\mathrm{pCO}_{2}$ data set from 14 open-ocean sites using a moored autonomous system. Earth Syst Sci Data. 2014;6:353-66.

34. Friederich GE, Brewer PG, Herlien R, Chavez FP. Measurement of sea surface partial pressure of $\mathrm{CO}_{2}$ from a moored buoy. Deep Sea Res Part I Oceanogr Res Pap. 1995;42:1175-86.

35. Sutton AJ, Feely RA, Sabine CL, McPhaden MJ, Takahashi T, Chavez FP, et al. Natural variability and anthropogenic change in equatorial Pacific surface ocean $\mathrm{pCO}_{2}$ and $\mathrm{pH}$. Glob Biogeochem Cycles. 2014;28:131-45.

36. Sutton AJ, Feely RA, Maenner-Jones S, Musielwicz S, Osborne J, Dietrich $\mathrm{C}$, et al. Autonomous seawater $\mathrm{pCO} 2$ and $\mathrm{pH}$ time series from 40 surface buoys and the emergence of anthropogenic trends. Earth Syst Sci Data Discuss. 2019;11:421-39.

37. Massaro RFS, de Carlo EH, Drupp PS, Mackenzie FT, Jones SM, Shamberger KE, et al. Multiple factors driving variability of $\mathrm{CO}_{2}$ exchange between the ocean and atmosphere in a tropical coral reef environment. Aquat Geochemistry. 2012;18:357-86. 
38. Drupp PS, De Carlo EH, Mackenzie FT, Sabine CL, Feely RA, Shamberger KE. Comparison of $\mathrm{CO}_{2}$ dynamics and Air-Sea gas exchange in differing tropical reef environments. Aquat Geochemistry. 2013;19:371-97.

39. Chatterjee A, Gierach MM, Sutton AJ, Feely RA, Crisp D, Eldering A, et al. Influence of El Niño on atmospheric $\mathrm{CO}_{2}$ over the tropical Pacific Ocean: findings from NASA's OCO-2 mission. Science. 2017;358:eaam5776.

40. Sutton AJ, Wanninkhof R, Sabine CL, Feely RA, Cronin MF, Weller RA. Variability and trends in surface seawater $\mathrm{pCO}_{2}$ and $\mathrm{CO}_{2}$ flux in the Pacific Ocean. Geophys Res Lett. 2017;44:5627-36.

41. Landschützer P, Gruber N, Bakker DCE, Schuster U, Nakaoka S, Payne MR, et al. A neural network-based estimate of the seasonal to inter-annual variability of the Atlantic Ocean carbon sink. Biogeosciences. 2013;10:7793-815.

42. Rödenbeck C, Keeling RF, Bakker DCE, Metzl N, Olsen A, Sabine C, et al. Global surface-ocean $\mathrm{pCO}_{2}$ and sea-air $\mathrm{CO}_{2}$ flux variability from an observation-driven ocean mixed-layer scheme. Ocean Sci. 2013;9:193-216.

43. Martz TR, Carr JJ, French CR, DeGrandpre MD. A submersible autonomous sensor for spectrophotometric $\mathrm{pH}$ measurements of natural waters. Anal Chem. 2003;75:1844-50.

44. Cullison Gray SE, DeGrandpre MD, Moore TS, Martz TR, Friederich GE, Johnson KS. Applications of in situ pH measurements for inorganic carbon calculations. Mar Chem. Elsevier B.V. 2011;125:82-90.

45. Fassbender AJ, Sabine CL, Lawrence-Slavas N, De Carlo EH, Meinig C, Maenner JS. Robust sensor for extended autonomous measurements of surface ocean dissolved inorganic carbon. Environ Sci Technol. 2015;49:3628-35.

46. Xue L, Cai WJ, Hu X, Sabine C, Jones S, Sutton AJ, et al. Sea surface carbon dioxide at the Georgia time series site (2006-2007): air-sea flux and controlling processes. Prog Oceanogr Elsevier Ltd. 2016;140:14-26.

47. Courtney TA, Andersson AJ, Bates NR, Collins A, Cyronak T, de Putron SJ, et al. Comparing chemistry and census-based estimates of net ecosystem calcification on a rim reef in Bermuda. Front Mar Sci. 2016;3:181.

48. Sandifer JR, Voycheck JJ. A review of biosensor and industrial applications of pH-ISFETs and an evaluation of Honeywell's "DuraFET". Microchim Acta. 1999;131:91-8.

49. Martz TR, Connery JG, Johnson KS. Testing the Honeywell Durafet for seawater $\mathrm{pH}$ applications. Limnol Oceanogr Methods. 2010;8:172-84.

50. Takeshita Y, Martz TR, Johnson KS, Dickson AG. Characterization of an ion sensitive field effect transistor and chloride ion selective electrodes for ph measurements in seawater. Anal Chem. 2014;86:11189-95.

51. Bresnahan PJ, Martz TR, Takeshita Y, Johnson KS, LaShomb M. Best practices for autonomous measurement of seawater $\mathrm{pH}$ with the Honeywell Durafet. Methods Oceanogr Elsevier Ltd. 2014;9: 44-60.

52. Johnson KS, Jannasch HW, Coletti LJ, Elrod VA, Martz TR, Takeshita Y, et al. Deep-Sea DuraFET: a pressure tolerant $\mathrm{pH}$ sensor designed for global sensor networks. Anal Chem. 2016;88:3249-56.

53. Gray JS. Marine biodiversity: patterns, threats and conservation needs. Biodivers Conserv Kluwer Academic Publishers. 1997;6: 153-75.

54. Costanza R, de Groot R, Sutton P, van der Ploeg S, Anderson SJ, Kubiszewski I, et al. Changes in the global value of ecosystem services. Glob Environ Chang Elsevier Ltd. 2014;26:152-8.

55. Hofmann GE, Smith JE, Johnson KS, Send U, Levin LA, Micheli F, et al. High-frequency dynamics of ocean $\mathrm{pH}$ : a multi-ecosystem comparison. Chin W-C, editor. PLoS One. 2011;6:e28983.
56. Johnson KS, Needoba JA, Riser SC, Showers WJ. Chemical sensor networks for the aquatic environment. Chem Rev. 2007;107:623-40.

57. Frieder CA, Nam SH, Martz TR, Levin LA. High temporal and spatial variability of dissolved oxygen and $\mathrm{pH}$ in a nearshore California kelp forest. Biogeosciences. 2012;9:3917-30.

58. Kapsenberg L, Hofmann GE. Ocean $\mathrm{pH}$ time-series and drivers of variability along the northern Channel Islands, California, USA. Limnol Oceanogr. 2016;61:953-68.

59. Gray SEC, DeGrandpre MD, Langdon C, Corredor JE. Short-term and seasonal $\mathrm{pH}, \mathrm{pCO}_{2}$ and saturation state variability in a coralreef ecosystem. Glob Biogeochem Cycles. 2012;26:1-13.

60. Takeshita Y, Cyronak T, Martz TR, Kindeberg T, Andersson. Coral reef carbonate chemistry variability at different functional scales. Front Mar Sci 2018;5:1-12.

61. Page HN, Courtney TA, De Carlo EH, Howins NM, Koester I, Andersson AJ. Spatiotemporal variability in seawater carbon chemistry for a coral reef flat in Kāne'ohe Bay, Hawai' i. Limnol Oceanogr. 2018; Ino.11084:1-22.

62. Guadayol Ò, Silbiger NJ, Donahue MJ, Thomas FIM, Silbiger NJ, Donahue MJ. Patterns in temporal variability of temperature, oxygen and $\mathrm{pH}$ along an environmental gradient in a coral reef. PLoS One. 2014;9:13104-12.

63. Saderne V, Fietzek P, Herman PMJ. Extreme variations of $\mathrm{pCO}_{2}$ and $\mathrm{pH}$ in a Macrophyte meadow of the Baltic Sea in summer: evidence of the effect of photosynthesis and local upwelling. PLoS One. 2013;8:2-9.

64. Pacella SR, Brown CA, Waldbusser GG, Labiosa RG, Hales B. Seagrass habitat metabolism increases short-term extremes and long-term offset of $\mathrm{CO}_{2}$ under future ocean acidification. Proc Natl Acad Sci. 2018;115:3870-5.

65. Mathis JT, Cross JN, Monacci N, Feely RA, Stabeno P. Evidence of prolonged aragonite undersaturations in the bottom waters of the southern Bering Sea shelf from autonomous sensors. Deep Res Part II Top Stud Oceanogr. Elsevier. 2014;109:125-33.

66. Harris KE, DeGrandpre MD, Hales B. Aragonite saturation state dynamics in a coastal upwelling zone. Geophys Res Lett. 2013;40: 2720-5.

67. Takeshita Y, Frieder CA, Martz TR, Ballard JR, Feely RA, Kram $\mathrm{S}$, et al. Including high-frequency variability in coastal ocean acidification projections. Biogeosciences. 2015;12:5853-70.

68. Price NN, Martz TR, Brainard RE, Smith JE. Diel variability in seawater $\mathrm{pH}$ relates to calcification and benthic community structure on coral reefs. PLoS One. 2012;7:1-9.

69. Frieder CA, Gonzalez JP, Bockmon EE, Navarro MO, Levin LA. Can variable $\mathrm{pH}$ and low oxygen moderate ocean acidification outcomes for mussel larvae? Glob Chang Biol. 2014;20:754-64.

70. Kapsenberg L, Miglioli A, Bitter MC, Tambutte E, Dumollard R, Gattuso J-P. Ocean pH fluctuations affect mussel larvae at key developmental transitions. Proc R Soc B Biol Sci. 2018;285: 20182381.

71. Comeau S, Edmunds PJ, Spindel NB, Carpenter RC. Diel $\mathrm{pCO}_{2}$ oscillations modulate the response of the coral Acropora hyacinthus to ocean acidification. Mar Ecol Prog Ser. 2014;501:99-111.

72. Small DP, Milazzo M, Bertolini C, Graham H, Hauton C, Hallspencer JM, et al. Sensitivity in natural systems. ICES J Mar Sci. 2016;73:604-12.

73. Kapsenberg L, Kelley AL, Shaw EC, Martz TR, Hofmann GE. Near-shore Antarctic $\mathrm{pH}$ variability has implications for the design of ocean acidification experiments. Sci Rep. 2015;5(9638):1-10.

74. Takeshita Y, McGillis W, Briggs EM, Carter AL, Donham EM, Martz TR, et al. Assessment of net community production and calcification of a coral reef using a boundary layer approach. J Geophys Res Ocean. 2016;121:5655-71.

75. Long MH, Charette MA, Martin WR, McCorkle DC. Oxygen metabolism and $\mathrm{pH}$ in coastal ecosystems: Eddy covariance 
hydrogen ion and oxygen exchange system (ECHOES). Limnol Oceanogr Methods. 2015;13:438-50.

76. Long MH, Nicholson DP. Surface gas exchange determined from an aquatic eddy covariance floating platform. Limnol Oceanogr Methods. 2018;16:145-59.

77. Waldbusser GG, Salisbury JE. Ocean acidification in the coastal zone from an organism's perspective: multiple system parameters, frequency domains, and habitats. Annu Rev Mar Sci. 2014;6:221-47.

78. Körtzinger A, Schimanski J, Send U. High quality oxygen measurements from profiling floats: a promising new technique. J Atmos Ocean Technol. 2005;22:302-8.

79. Gruber N, Doney SC, Emerson SR, Gilbert D, Kobayashi T, Körtzinger A, et al. Adding oxygen to Argo: developing a global in-situ observatory for ocean deoxygenation and biogeochemistry. Paper presented at Ocean Obs '09. 2009.

80. Johnson KS, Coletti LJ, Jannasch HW, Sakamoto CM, Swift DD, Riser SC. Long-term nitrate measurements in the ocean using the in situ ultraviolet spectrophotometer: sensor integration into the APEX profiling float. J Atmos Ocean Technol. 2013;30:1854-66.

81. Boss E, Swift D, Taylor L, Brickley P, Zaneveld R, Riser S, et al. Observations of pigment and particle distributions in the western from North Atlantic an autonomous float and ocean color satellite. Limnol Oceanogr. 2008;53:2112-22.

82. Sullivan JM, Twardowski MS, Ronald J, Zaneveld V, Moore CC. Measuring optical backscattering in water. Light Scatt Rev 7. Berlin: Springer Berlin, Heidelberg; 2013. p. 189-224.

83. Riser SC, Johnson KS. Net production of oxygen in the subtropical ocean. Nature. 2008;451:323-6.

84. Plant JN, Johnson KS, Sakamoto CM, Jannasch HW, Coletti LJ, Riser SC, et al. Net community production at Ocean Station Papa observed with nitrate and oxygen sensors on profiling floats. Glob Biogeochem Cycles. 2016;30:859-79.

85. Martz TR, Johnson KS, Riser SC. Ocean metabolism observed with oxygen sensors on profiling floats in the south pacific. Limnol Oceanogr. 2008;53:2094-111.

86. Bushinsky SM, Emerson SR. Marine biological production from in situ oxygen measurements on a profiling float in the subarctic Pacific Ocean. Glob Biogeochem Cycles. 2015;29:2050-60.

87. Yang B, Emerson SR, Bushinsky SM. Annual net community production in the subtropical Pacific Ocean from in-situ oxygen measurements on profiling floats. Glob Biogeochem Cycles. 2017:31:728-44.

88. Johnson KS, Riser SC, Karl DM. Nitrate supply from deep to near-surface waters of the North Pacific subtropical gyre. Nature. 2010;465:1062-5.

89. Johnson KS, Riser SC, Ravichandran M. Oxygen variability controls denitrification in the bay of Bengal oxygen minimum zone. Geophys Res Lett. 2019:1-8.

90. Bushinsky SM, Gray AR, Johnson KS, Sarmiento JL. Oxygen in the Southern Ocean from Argo floats: determination of processes driving air-sea fluxes. J Geophys Res Ocean 2017;122:1-22.

91. Bushinsky SM, Emerson SR. Biological and physical controls on the oxygen cycle in the Kuroshio Extension from an array of profiling floats. Deep Sea Res Part I Oceanogr Res Pap. 2018;141:51-70.

92. Wolf MK, Hamme RC, Gilbert D, Yashayaev I, Thierry V. Oxygen saturation surrounding deep water formation events in the Labrador Sea from Argo-O 2 data. Glob Biogeochem Cycles. 2018;32:635-53.

93. Fawcett SE, Johnson KS, Riser SC, Van Oostende N, Sigman DM. Low-nutrient organic matter in the Sargasso Sea thermocline: a hypothesis for its role, identity, and carbon cycle implications. Mar Chem Elsevier. 2018;207:108-23.
94. Fiedler B, Fietzek P, Vieira N, Silva P, Bittig HC, Körtzinger A. In situ $\mathrm{CO}_{2}$ and $\mathrm{O}_{2}$ measurements on a profiling float. J Atmos Ocean Technol Am Meteorological Soc. 2013;30:112-26.

95. Johnson KS, Plant JN, Coletti LJ, Jannasch HW, Sakamoto CM, Riser SC, et al. Biogeochemical sensor performance in the SOCCOM profiling float array. J Geophys Res Ocean. 2017;122:6416-36.

96. Gruber N, Landschützer P, Lovenduski NS. The variable Southern Ocean carbon sink. Annu Rev Mar Sci. 2019;11:1-28.

97. Sarmiento JL, Gruber N, Brzezinski MA, Dunne JP. High-latitude controls of thermocline nutrients and low latitude biological productivity. Nature. 2004;427:56-60.

98. Williams NL, Juranek LW, Feely RA, Johnson KS, Sarmiento JL, Talley LD, et al. Calculating surface ocean $\mathrm{pCO}_{2}$ from biogeochemical Argo floats equipped with $\mathrm{pH}$ : an uncertainty analysis. Glob Biogeochem Cycles. 2017;31:591-604.

99. Williams NL, Juranek LW, Feely RA, Russell JL, Johnson KS, Hales B. Assessment of the carbonate chemistry seasonal cycles in the Southern Ocean from persistent observational platforms. J Geophys Res Ocean. 2018;123:4833-52.

100. Gray AR, Johnson KS, Bushinsky SM, Riser SC, Russell JL, Talley LD, et al. Autonomous biogeochemical floats detect significant carbon dioxide outgassing in the high-latitude Southern Ocean. Geophys Res Lett. 2018;45:9049-57.

101. Fay AR, Lovenduski NS, McKinley GA, Munro DR, Sweeney C, Gray AR, et al. Utilizing the Drake Passage time-series to understand variability and change in subpolar Southern Ocean $\mathrm{pCO}_{2}$ Biogeosci Discuss. 2017;15:1-31.

102. Briggs EM, Martz TR, Talley LD, Mazloff MR, Johnson KS. Physical and biological drivers of biogeochemical tracers within the seasonal sea ice zone of the Southern Ocean from profiling floats. J Geophys Res Ocean. 2018;123:746-58.

103. Fassbender AJ, Bourbonnais A, Clayton S, Gaube P, Omand M, Franks PJS, et al. Interpreting mosaics of ocean biogeochemistry. Eos, 99, https://doi.org/10.1029/2018EO109707.

104. Feely RA, Sabine CL, Hernandez-Ayon JM, Ianson D, Hales B. Evidence for upwelling of corrosive "acidified" water onto the continental shelf. Science. 2008;320:1490-2.

105. Chan F, Barth JA, Blanchette CA, Byrne RH, Chavez F, Cheriton $\mathrm{O}$, et al. Persistent spatial structuring of coastal ocean acidification in the California current system. Sci Rep. 2017;7:1-7.

106. Ohman M, Rudnick D, Chekalyuk A, Davis R, Feely R, Kahru M, et al. Autonomous ocean measurements in the California current ecosystem. Oceanography. 2013;26:18-25.

107. Alin SR, Feely RA, Dickson AG, Hernández-Ayón JM, Juranek LW, Ohman MD, et al. Robust empirical relationships for estimating the carbonate system in the southern California current system and application to CalCOFI hydrographic cruise data (20052011). J Geophys Res. 2012;117:C05033.

108. Wulff T, Lehmenhecker S, Bauerfeind E, Hoge U, Shurn K, Klages M. Biogeochemical research with an autonomous underwater vehicle: payload structure and arctic operations. In OCEANS-Bergen, 2013 MTS/IEEE. IEEE pp. 1-10.

109. Hemming MP, Kaiser J, Heywood KJ, Bakker DCE, Boutin J, Shitashima $\mathrm{K}$, et al. Measuring $\mathrm{pH}$ variability using an experimental sensor on an underwater glider. Ocean Sci. 2017;13:427-42.

110. Saba GK, Wright-fairbanks E, Miles TN, Chen B, Cai W, Wang K, et al. Developing a profiling glider $\mathrm{pH}$ sensor for high resolution coastal ocean acidification monitoring. In Ocean 2018 MTS/IEEE Charleston, 2018. IEEE pp.1-8.

111. Hine R, Willcox S, Hine G, Richardson T. The wave glider: a wave-powered autonomous marine vehicle. Ocean 2009, MTS/ IEEE Biloxi - Mar Technol Our Futur Glob Local Challenges. IEEE. 2009;6:1-6.

112. Cokelet ED, Meinig C, Lawrence-Slavas N, Jenkins R, Mordy CW, Tabisola HM, et al. The use of Saildrones to examine spring 
conditions in the Bering Sea: vehicle specification and mission performance. In Ocean 2015 - MTS/IEEE Washington, 2015. MTS, pp. 1-6.

113. Chavez FP, Sevadjian J, Wahl C, Friederich J, Friederich GE. Measurements of $\mathrm{pCO}_{2}$ and $\mathrm{pH}$ from an autonomous surface vehicle in a coastal upwelling system. Deep Res Part II Top Stud Oceanogr. Elsevier. 2018;151:137-46.

114. Mordy CW, Cokelet ED, De Robertis A, Jenkins R, Kuhn CE, Lawrence-Slavas N, et al. Advances in ecosystem research: Saildrone surveys of oceanography, fish, and marine mammals in the Bering Sea. Oceanography. 2017;30:113-5.

115. Clarke JS, Achterberg EP, Connelly DP, Schuster U, Mowlem M. Developments in marine $\mathrm{pCO}_{2}$ measurement technology; towards sustained in situ observations. TrAC - Trends Anal Chem. Elsevier Ltd. 2017;88:53-61.

116. Fritzsche E, Staudinger C, Fischer JP, Thar R, Jannasch HW, Plant $\mathrm{JN}$, et al. A validation and comparison study of new, compact, versatile optodes for oxygen, $\mathrm{pH}$ and carbon dioxide in marine environments. Mar Chem. 2018;207:63-76.

117. Liu X, Byrne RH, Adornato L, Yates KK, Kaltenbacher E, Ding X, et al. In situ spectrophotometric measurement of dissolved inorganic carbon in seawater. Environ Sci Technol. 2013;47:11106-14.

118. Wang ZA, Sonnichsen FN, Bradley AM, Hoering KA, Lanagan $\mathrm{TM}$, Chu SN, et al. In situ sensor technology for simultaneous spectrophotometric measurements of seawater total dissolved inorganic carbon and pH. Environ Sci Technol. 2015;49:4441-9.

119. Chu SN, Wang ZA, Gonneea ME, Kroeger KD, Ganju NK. Deciphering the dynamics of inorganic carbon export from intertidal salt marshes using high-frequency measurements. Mar Chem. Elsevier. 2018;206:7-18

120. Spaulding RS, Degrandpre MD, Beck JC, Hart RD, Peterson B, De Carlo EH, et al. Autonomous in situ measurements of seawater alkalinity. Environ Sci Technol. 2014;48:9573-81.

121. Rérolle VMC, Floquet CFA, Harris AJK, Mowlem MC, Bellerby RRGJ, Achterberg EP. Development of a colorimetric microfluidic $\mathrm{pH}$ sensor for autonomous seawater measurements. Anal Chim Acta. Elsevier B.V. 2013;786:124-31.

122. Briggs EM, Sandoval S, Erten A, Takeshita Y, Kummel AC, Martz TR. Solid state sensor for simultaneous measurement of total alkalinity and $\mathrm{pH}$ of seawater. ACS Sensors. 2017;2:1302-9.

123. Afshar MG, Crespo GA, Bakker E. Thin-layer chemical modulations by a combined selective proton pump and $\mathrm{pH}$ probe for direct alkalinity detection. Angew Chemie - Int Ed. 2015;54:8110-3.

124. Sharp JD, Byrne RH, Liu X, Feely RA, Cuyler EE, Wanninkhof R, et al. Spectrophotometric determination of carbonate ion concentrations: elimination of instrumentdependent offsets and calculation of in situ saturation states. Environ Sci Technol. 2017;51:9127-36.

125. Sharp JD, Byrne RH. Carbonate ion concentrations in seawater: spectrophotometric determination at ambient temperatures and evaluation of propagated calculation uncertainties. Mar Chem. Elsevier. 2018:1-11.

126. Easley RA, Patsavas MC, Byrne RH, Liu X, Feely RA, Mathis JT. Spectrophotometric measurement of calcium carbonate saturation states in seawater. Environ Sci Technol. 2013;47:1468-77.

127. McLaughlin K, Nezlin NP, Weisberg SB, Dickson AG, Booth JA, Cash CL, et al. An evaluation of potentiometric pH sensors in coastal monitoring applications. Limnol Oceanogr Methods. 2017;15:679-89.

128. McLaughlin K, Weisberg S, Dickson A, Hofmann G, Newton J, Aseltine-Neilson D, et al. Core principles of the California current acidification network: linking chemistry, physics, and ecological effects. Oceanography. 2015;25:160-9.
129. Waldbusser GG, Hales B, Haley BA. Calcium carbonate saturation state: on myths and this or that stories. ICES J Mar Sci J Cons. 2016;73:563-8.

130. Bednaršek N, Tarling GA, Bakker DCE, Fielding S, Jones EM, Venables HJ, et al. Extensive dissolution of live pteropods in the Southern Ocean. Nat Geosci Nature Publishing Group. 2012;5:881-5.

131. Berelson WM, Balch WM, Najjar R, Feely RA, Sabine C, Lee K. Relating estimates of $\mathrm{CaCO}_{3}$ production, export, and dissolution in the water column to measurements of $\mathrm{CaCO}_{3}$ rain into sediment traps and dissolution on the sea floor: a revised global carbonate budget. Glob Biogeochem Cycles. 2007;21:1-15.

132. Estapa ML, Buesseler K, Boss E, Gerbi G. Autonomous, highresolution observations of particle flux in the oligotrophic ocean. Biogeosciences. 2013;10:5517-31.

133. Bishop JKB, Fong MB, Wood TJ. Robotic observations of high wintertime carbon export in California coastal waters. Biogeosciences. 2016;13:3109-29.

134. Wong A, Keeley R, Carval T. Argo quality control manual, v2.32. Argo Data Management. 2009;1-50.

135. Williams NL, Juranek LW, Johnson KS, Feely RA, Riser SC, Talley LD, et al. Empirical algorithms to estimate water column pH in the Southern Ocean. Geophys Res Lett. 2016;43:3415-22.

136. Carter BR, Feely RA, Williams NL, Dickson AG, Fong MB, Takeshita Y. Updated methods for global locally interpolated estimation of alkalinity, $\mathrm{pH}$, and nitrate. Limnol Oceanogr Methods. 2018;16:119-31.

137. Sauzède R, Bittig HC, Claustre H, Pasqueron de Fommervault $\mathrm{O}$, Gattuso J-P, Legendre L, et al. Estimates of water-column nutrient concentrations and carbonate system parameters in the global ocean: a novel approach based on neural networks. Front Mar Sci. 2017;4:1-17.

138. Bittig HC, Steinhoff T, Claustre H, Fiedler B, Williams NL, Sauzède $\mathrm{R}$, et al. An alternative to static climatologies: robust estimation of open ocean $\mathrm{CO}_{2}$ variables and nutrient concentrations from $\mathrm{T}, \mathrm{S}$, and $\mathrm{O}_{2}$ data using Bayesian neural networks. Front Mar Sci. 2018;5:328.

139. Lai C, Degrandpre MD, Darlington RC. Autonomous optofluidic chemical analyzers for marine applications: insights from the submersible autonomous moored instruments ( SAMI ) for $\mathrm{pH}$ and $\mathrm{p}$ CO 2. Front Mar Sci. 2018;4:1-11.

140. Takeshita Y, Johnson KS, Martz TR, Plant JN, Sarmiento JL. Assessment of autonomous $\mathrm{pH}$ measurements for determining surface seawater partial pressure of $\mathrm{CO}_{2}$. J Geophys Res Ocean. 2018;123:4003-13.

141. Orr JC, Epitalon JM, Dickson AG, Gattuso JP. Routine uncertainty propagation for the marine carbon dioxide system. Mar Chem. Elsevier. 2018;207:84-107.

142. Chen B, Cai WJ, Chen L. The marine carbonate system of the Arctic Ocean: assessment of internal consistency and sampling considerations, summer 2010. Mar Chem. Elsevier B.V. 2015;176:174-88.

143. Müller JD, Bastkowski F, Sander B, Seitz S, Turner DR, Dickson $\mathrm{AG}$, et al. Metrology for $\mathrm{pH}$ measurements in brackish waterspart 1: extending electrochemical pHT measurements of TRIS buffers to salinities 5-20. Front Mar Sci. 2018;5:1-12.

144. Cai W-J, Wang Y, Hodson RE. Acid-Base properties of dissolved organic matter in the estuarine waters of Georgia, USA. Geochim Cosmochim Acta. 1998;62:473-83.

145. Yang B, Byrne RH, Lindemuth M. Contributions of organic alkalinity to total alkalinity in coastal waters: a spectrophotometric approach. Mar Chem. Elsevier B.V. 2015;176:199-207.

146. Fong MB, Dickson AG. Insights from GO-SHIP hydrography data into the thermodynamic consistency of $\mathrm{CO}_{2}$ system measurements in seawater. Mar. Chem. Elsevier B.V. 2019;211:52-63. 
147. Carter BR, Williams NL, Gray AR, Feely RA. Locally interpolated alkalinity regression for global alkalinity estimation. Limnol Oceanogr Methods. 2016;14:268-277.

148. Talley LD, Rosso I, Kamenkovich I, Mazloff MR, Wang J, Boss E, et al. Southern Ocean biogeochemical float deployment strategy, with example from the Greenwich Meridian Line (GO-SHIP A12). J Geophys Res Ocean. 2019;124:403-31.

149. Majkut JD, Carter BR, Frolicher TL, Dufour CO, Rodgers KB, Sarmiento JL. An observing system simulation for Southern Ocean carbon dioxide uptake. Philos Trans R Soc A Math Phys Eng Sci. 2014;372:20130046.

150. Kamenkovich I, Haza A, Gray AR, Dufour CO, Garraffo Z. Observing system simulation experiments for an array of autonomous biogeochemical profiling floats in the Southern Ocean. J Geophys Res Ocean. 2017;122:7595-611.

151. Verdy A, Mazloff MR. A data assimilating model for estimating Southern Ocean biogeochemistry. J Geophys Res Ocean. 2017;122:2017-33.
152. Russell JL, Kamenkovich I, Bitz C, Ferrari R, Gille ST, Goodman PJ, et al. Metrics for the evaluation of the Southern Ocean in coupled climate models and earth system models. J Geophys Res Ocean. 2018;123:3120-43.

153. Alexander G. Vincent, Robin W. Pascal, Alexander D. Beaton, John Walk, Joanne E. Hopkins, E. Malcolm S. Woodward, Matthew Mowlem, Maeve C. Lohan, (2018) Nitrate drawdown during a shelf sea spring bloom revealed using a novel microfluidic in situ chemical sensor deployed within an autonomous underwater glider. Marine Chemistry 205:29-36

Publisher's Note Springer Nature remains neutral with regard to jurisdictional claims in published maps and institutional affiliations. 